\title{
Retraction Note to: Silencing circular RNA circ_0010729 protects human cardiomyocytes from oxygen-glucose deprivation-induced injury by up-regulating microRNA-145-5p
}

\author{
Qifeng Jin ${ }^{1} \cdot$ Yuanyuan Chen ${ }^{2}$ (D)
}

Published online: 12 January 2022

๑) Springer Science+Business Media, LLC, part of Springer Nature 2022
Publisher's Note Springer Nature remains neutral with regard to jurisdictional claims in published maps and institutional affiliations.

The Editors in Chief have retracted this article after an investigation by The Chinese Ministry of Science and Technology into this article brought to light evidence of research misconduct. The Editors in Chief no longer have confidence in the presented results and the conclusions drawn in this study. Qifeng Jin and Yuanyuan Chen have not responded to any correspondence from the editor or publisher about this retraction.

The original article can be found online at https://doi.org/10.1007/ s11010-019-03621-9.

Yuanyuan Chen

yuanyuan25c@163.com

1 Department of Cardiology I, Affiliated Hospital of Jining Medical University, Jining 272000, China

2 Department of Cardiology V, Affiliated Hospital of Jining Medical University, No. 89 Guhuai Road, Jining 272000, Shandong, China 